\title{
A cross-sectional analysis of the factors that shape adult nursing students' values,
} attitudes and perceptions of compassionate care

\begin{abstract}
Nursing students are the future of nursing and are situated between the expectation of the public: that they will receive compassionate care, and the profession: that it will continue to develop technically to meet the needs of the changing healthcare economy. Research has focused on the factors affecting registered nurses' values in care, however, less is known regarding the factors that shape nursing students' values, attitudes, and perceptions of compassionate care.
\end{abstract}

Six focus group discussions with 23 adult branch nursing students (years 1-3) from a United Kingdom (UK) university were conducted to ascertain their understanding of personal and professional values, factors influencing these values, and whether they felt these values influenced the way they provided compassionate care?

Qualitative thematic analysis resulted in 5 themes, 3 of which were the focus of this paper: general values, fundamental nursing and nursing values. Participants considered a range of influencers of their general values, and demonstrated a transparency between their personal and professional values, with respect and 
dignity being important. Fundamental nursing was complex to consider, but was considered to orientate around the provision of care in a compassionate way.

\section{Keywords}

Values, care and caring, compassion, nursing students, qualitative research, crosssectional design 


\section{Introduction}

Fundamental nursing values

In recent years, particularly in the UK, nurses have been accused of no longer caring, which has been reported as a perceived loss of compassion, dignity and respect for the person (Care Quality Commission 2015; Francis, 2013; Keogh, 2013). This has prompted debate about what constitutes fundamental nursing values, and how these, or a lack of them, might impact upon the provision of good nursing care (Biro, 2012). Horton et al. (2007) consider the notion of the 'good nurse' as inseparable from values constructing nursing practice; indeed 'good nursing' is defined by its values. Nursing values signify what is considered important both of the value of nursing and values within nursing (Horton et al., 2007). Their findings span eight areas, including for example, nursing being synonymous with caring for the sick, and caring as a way of knowing the patient. However, they mention that culture and personal values influences nursing values.

Horton et al. (2007) suggests the advancement of clinical nursing roles are ascribed greater importance in society, compared with the focus on fundamental nursing care. In exploring what nursing care is and whether it differs from care in nursing, Pearcey (2010) highlights caring appears an extra aspect to the functional tasks nurses undertake; a quality of the nurses' demeanour rather than the task they 
perform. Caring pertains to the "little things that [nurses] are not supposed to do anymore" (Pearcey, 2010, p. 53), either because of insufficient time to sit and talk with a patient, or because other tasks need completing, which leads to whether caring itself might be perceived as a "devaluing burden delegated to healthcare assistants" (Pearcey, 2010, p. 54). Subsequent negative impacts on the professional fulfilment of nurses is likely, with Perry (2005) finding nurses derive professional fulfilment when they are able to affirm to patients that they are cared about; advocate for patients when not able to themselves; create, facilitate, and share feelings of hope with patients; and help patients to find meaning in their illness or experience.

Internationally, Pang et al. (2009) studied registered nurses suggesting altruism, caring, trustworthiness, dignity, developing the profession, autonomy, and justice are key nursing values in China. Whilst in Taiwan, nursing values pertain to humanistic care, professionally competent and holistic care, fostering growth and discovering life meaning, the 'give and take' of caring for others, financial compensation, and raising public health (Shih et al., 2008).

Chipman (1991) has identified nursing students' notions of the value of caring being the giving of themselves in meeting patients' needs and providing comfort. Furthermore, Kelly (1991) reported nursing students understood 'professional 
values' as having respect for others and taking time for 'little things', for example, looking for dentures. These altruistic notions of care differentiate the values of nursing students to other student groups, such as management students, who score lower on altruism, but higher on autonomy and risk (Thorpe and Loo, 2003).

Good nurses who are good people

Since the time of Florence Nightingale, good nursing care depends on a historical ideal of good nurses who are good people, demonstrating a compassionate quality of their character (Bradshaw, 2011). Compassion refers to 'personal qualities' (Bradshaw, 2011, p. 13) of the nurse, which are then 'nurtured in, and by, the culture and ethos of clinical practice' (Bradshaw, 2011, p. 14). This represents the symbiotic relationship between Higher Education Institutions (HEIs), providing the academic (scientific) programme, and clinical practice, which provides exposure to the reality of the practice (art) of nursing. Furthermore, nurse educators expect student nurses to demonstrate compassion upon entry to nurse training (Curtis et al., 2012; Morris-Thompson, 2012), and for compassion to be assessed for registered nurses during their appraisals and recruitment interviews (Dean, 2012).

Compassion is considered a fundamental aspect of nursing, part of the essence of providing care to others (Bramley and Matiti, 2014). However, compassion is not a 
unidimensional concept but rather, 'a process of intuition and communication' (van der Cingel 2014, p. 1255), recognised through attentiveness, active listening, naming of suffering, nurse involvement with the patient's situation, helping, being present (consciously alert), and understanding suffering.

$6 C s$

In the UK, the Chief Nursing Officer (CNO) implemented a strategy (Department of Health (DH), 2012a) referred to as the 6Cs representing: care, compassion, competence, communication, courage, and commitment. This strategy developed from a consultation (DH, 2012b) eliciting what the 6Cs meant to nurses, midwives, and carers, and what was needed to embed these values and behaviours into care. However, Bradshaw (2015) highlights several issues with the CNOs strategy, with no reference to international nursing literature discussing the philosophy of nursing, and that M. Simone Roach's (1984, 1992, as cited in Bradshaw, 2015) work identifying 6Cs (compassion, competence, confidence, conscience, commitment, comportment) is not mentioned.

Rationale 
Much of the existing research examining nursing values, attitudes and perceptions of compassionate care focuses on registered nurses, rather than nursing students. Of the 32 studies reviewed by Horton et al. (2007), only 6 involved a sample of nursing students. Similarly, Pang et al. (2009); Pearcey (2010); Perry (2005); and Shih et al. (2008) have focused on registered nurses' values and perceptions of compassionate care. For pre-registration nursing programmes to prepare nursing students to deliver compassionate care alongside balancing competing demands (Horsburgh and Ross, 2013), it is important to determine factors that shape nursing students' values, attitudes and perceptions of compassionate care. Such findings may help nursing students reflect on the notion of compassionate care and nurse educators develop curricula that nurture nursing students' development and application of compassion in practice.

Aims

1. Ascertain pre-registration adult branch nursing students' understanding of personal and professional values.

2. Identify factors that shaped their personal and professional values.

3. Explore whether personal and professional values influence their ability to provide compassionate care. 


\section{Methodology}

Design

A cross-sectional qualitative design was utilised comprising six focus group interviews. The cross-sectional design enabled the collection of data from nursing students across year groups 1-3 at the point in time of their study. Focus group interviews were particularly useful, rather than one-one interviews, to help participants feel comfortable amongst their peers. This in turn enabled the facilitator(s) to understand how participants' statements were challenged, extended, developed, undermined, or qualified in light of discussions (Willig, 2008).

\section{Ethics and recruitment}

University ethical clearance was obtained through the Faculty of Health Sciences Research Ethics Committee proportionate review. Nursing students were assured their participation was voluntary and that they could withdraw from the study at any point without their nurse education being affected. Nursing students were recruited into the study firstly by an email, containing an invitation letter (with dates of the interviews) and information sheet sent by programme leaders to all pre-registration adult nursing students within the Faculty. Nursing students were given time to consider the information and asked to notify the named member of the research team identified of their intention to join a focus group interview. 


\section{Participants}

A total of 23 pre-registration adult branch nursing students across years 1, 2, and 3 participated (Table 1). All participants were of white British ethnicity. Participants in year one of study comprised focus groups $2(n=5)$ and $5(n=6)$; year two of study comprised focus groups $1(n=2)$ and $6(n=4)$; and year 3 of study comprised focus groups $3(n=2), 4(n=3)$ and $6(n=1)$. All were undertaking their programme of nurse education at a university in the West Midlands in the UK, in an inner city area with clinical placements in both urban and rural areas.

Table 1

Demographic profile of participants

\begin{tabular}{lllll}
\hline Year & \multicolumn{1}{c}{ One } & \multicolumn{1}{c}{ Two } & \multicolumn{1}{c}{ Three } & \multicolumn{1}{c}{ Total } \\
\hline $\begin{array}{l}\text { Number of } \\
\text { participants }\end{array}$ & 11 & 6 & 6 & 23 \\
$\begin{array}{l}\text { Age range } \\
\text { Gender }\end{array}$ & $\begin{array}{l}\text { 28-44 years } \\
\text { 8 Female }\end{array}$ & $\begin{array}{l}\text { 21-30 years } \\
\text { 6 Female }\end{array}$ & $\begin{array}{l}\text { 22-48 years } \\
\text { 4 Female }\end{array}$ & $\begin{array}{l}\text { 21-48 years } \\
\text { 18 Female } \\
\text { 2 Male }\end{array}$ \\
\hline
\end{tabular}

Data collection 
Between March-October 2014, data was collected through a series of 6 focus group interviews. Focus groups lasted between 37 minutes and 1 hour 7 minutes $($ mean $=$ 52 minutes) and were semi-structured with an interview schedule to facilitate open discussion between participants. The interview schedule initially focused on what participants understood by the term values, including their personal and professional (nursing) values. This was followed by questions exploring how they would apply their values in clinical practice identifying factors pertinent in shaping their values. Questions were used to explore whether participants' values influenced their ability to provide compassionate care, and of their thoughts on fundamental nursing and differences between care and caring.

\section{Data analysis}

Focus group audio recordings were transcribed verbatim by an independent professional transcriber, and then subjected to inductive thematic analysis (Braun and Clarke, 2006). This qualitative method was used to identify, analyse, and report patterns and themes within the data, focusing on themes that were strongly evidenced by the data itself (Patton, 1990). This took place in six phases (Table 2).

Table 2 
Inductive thematic analysis phases

\begin{tabular}{ll}
\hline Phase & \multicolumn{1}{c}{ Activity } \\
\hline 1 & $\begin{array}{l}\text { Reading and re-reading of the transcript and initial notation } \\
\text { (familiarisation with data) }\end{array}$ \\
2 & $\begin{array}{l}\text { Generation of initial codes (noting interesting features systematically) } \\
\text { Searching for themes based on initial codes (collation of codes into } \\
\text { potential themes) }\end{array}$ \\
4 & $\begin{array}{l}\text { Reviewing themes (analysis of whether themes correspond to codes) } \\
\text { Defining and development of themes (further detailed analysis and } \\
\text { general collation) }\end{array}$ \\
6 & $\begin{array}{l}\text { Reporting and dissemination (final analyses and collation of themes for } \\
\text { reporting) }\end{array}$ \\
\hline
\end{tabular}

\section{Results}

The thematic analysis resulted in the development of five broad themes: (1) general values (2) nursing values (3) fundamental nursing (4) stress management and (5) nurse education. The focus of this manuscript is on fundamental nursing, and nursing values (see Figure 1 for Thematic Map) with a specific emphasis upon the implications of these findings for the provision of compassionate person-centred care.

Figure 1

Thematic map of superordinate themes and themes

Insert Figure 1 
Of these 3 superordinate themes and 6 themes, a further 20 subthemes emerged (Table 3) illustrating the complexity by which participants understood values in general, how they pertained to nursing, and how they translated in the way nursing care is provided?

Table 3

Themes and subthemes

\begin{tabular}{|c|c|}
\hline $\begin{array}{c}\text { Superordinate } \\
\text { Themes }\end{array}$ & Themes and Subthemes \\
\hline General values & $\begin{array}{l}\text { Influencers: culture; childhood upbringing; religion; } \\
\text { education; life experience; legislation; media. } \\
\text { Personal values: transparency between personal and } \\
\text { professional values; ideal behaviours; respect and } \\
\text { dignity. }\end{array}$ \\
\hline Fundamental nursing & $\begin{array}{l}\text { Difficult to determine: different opinions. } \\
\text { 'Whatever the patient needs': care; compassion. } \\
\text { Nursing demeanour: 'care' and 'caring'. }\end{array}$ \\
\hline Nursing values & $\begin{array}{l}\text { 6Cs: } \\
\text { care; } \\
\text { commitment. }\end{array}$ \\
\hline
\end{tabular}

The thematic analysis demonstrated a interconnectedness between all themes, with influencers of general values translating into personal values, which were then transferred tacitly into nursing values, providing the foundation by which participants understood notions of fundamental nursing. 
General values

This superordinate theme refers to the broad range of factors participants felt shaped their own particular values. These factors were both intrinsic and extrinsic, for example, originating either personally, culturally, or societally. The subtheme, influencers, is used to delineate these factors indicating how they shaped participants' attitudes, beliefs, and behaviours before entering nurse education. It is clear that some participants entered their programme of study with some very entrenched values, illustrated through the theme personal values.

Personal values: Respect and dignity. Many participants recognised this as an essential component of their own core values, affirming how this is essential in nurturing self-worth and respect for others:

'[Values depend] on what you believe, so your belief when you are a nurse, when you are on that ward and you are dealing with those patients [...] you want them to be dealt with in a nice respectful way' (FG2, P5, L12).

The demonstration of being 'nice' and 'respectful' was through the participant being polite and having a common courtesy: 
'Being nice to people, it is not hard to give a smile in the morning [and] say “hello".' (FG6, P4, L43).

Participants also demonstrated an awareness of the ethics of reciprocity, for example The Golden Rule, as expressed in the following:

'YYou] gain a respect for other people because of the way they have respected you.' (FG3, P2, L10).

This positive or direct application of the ethic of reciprocity, as opposed to the negative application in not treating others in ways that one would not like to be treated, illustrates the interdependency of respect (as deep admiration and due regard for another's feelings) and reciprocity (as exchange for mutual benefit). However, this ethic goes beyond demonstrating respect solely for mutual benefit, as highlighted in the following, where there might be discordance between one's own personal values and those of others:

'[Values include] respecting people's opinions and like their values as well not just thinking about your own values, respecting like other people's ideas and what they believe in.' (FG4, P3, L13). 
The majority of participants used values of respect and dignity as a foundation, a benchmark that informed their own attitudes, behaviours and actions. This transferred into how participants perceived the themes of fundamental nursing.

\section{Fundamental nursing}

The notion of fundamental nursing created ambiguity and contradiction for participants given the variety of opinions nursing students, registered nurses and the multidisciplinary team, would have towards nursing. However, participants considered fundamental nursing referred to the notion of care and compassion to fulfill the needs of the patient and enhance their well-being.

'Whatever the patient needs': Care: Participants reported that 'care' was the core value associated with fundamental nursing, centred on seeing the humanity of the person. The humanity of the person was preserved by a wide range of nursing activities involving both instrumental aspects of care and affective approaches such as active listening, ensuring care was person-centred, and meeting the personal needs of the individual to:

'[...] care for a patient, be able to make them feel human, for example, if they 
are laying in a bed and they have soil[ed] themselves, then changing the bed and making them get some new clothes on, it just makes them feel human' (FG1, P1, L69).

There was recognition that care should be holistic and meet the needs of the patient's family and friends:

'[...] not just [for] the patient, it's their family, friends, whoever [...] assessing them as a whole' (FG1, P2, L76).

Holistic care involved 'knowing the patient' (seeing the individual) and establishing a relationship of trust:

'[...] to see an individual in a patient, not just a patient [...] and not a number [...] just showing people that you see them as individuals and care for them as individual, for that very person, for their preferences and likes and dislikes.' (FG2, P4, L119, 121, 123).

Participants acknowledged that care was about caring for someone in their time of need or vulnerability, supporting them across the lifespan trajectory either to recovery or a comfortable death: 
'[Fundamental nursing] boils down to a person who cares for someone else in their time of need, when they can't care for themselves [...] whether or not they're poorly, or whether or not you're helping them get better, or helping them have a comfortable death' (FG3, P2, L51).

Interestingly, while participants made frequent reference to holistic care in some of their descriptions of the concept, they did not include specific reference to the spiritual dimension:

'[Fundamental nursing care includes] physical, social, and psychological aspects of well-being [...] the full circle definitely so rather than just the functional needs [...] attending to the social and the psychological issues as well' (FG3, P1, L138).

When asked regarding spiritual care and fundamentals of nursing, one participant highlighted that spiritual needs may be "forgotten" whilst concentrating on other patient needs:

'[Nursing is] very physical [...] attend to the physical needs really well [...] we definitely forget about the spiritual needs [...] along with the psychological 
needs [...] we're very task [orientated].' (FG3, P2, L147).

Fundamental nursing as care was described as the standard of care one would expect themselves and also closely linked to patient safety:

'[Fundamental nursing is] about providing the level of care you would expect for yourself, or a relative or loved on, and if you're giving out care that is below that standard, or below what you would expect, it's not good enough.' (FG4, P1, L91).

'[Fundamental nursing implies] keeping patients safe [...] working within your capabilities and seeking assistance if you're not sure.' (FG4, P2, L94).

However, ambiguity remained with one participant highlighting that fundamental nursing (in its various forms) has impact but cannot be easily measured:

'[Fundamental nursing] adds a lot of value to people's lives that isn't necessarily measured' (FG3, P1, L156).

'Whatever the patient needs': Compassion. Participant's implied that there was a mutual relationship between dignity and compassion: 
[...] treating [the patient] with dignity and upholding their values, because I think dignity can be defined [...] but I think dignity is the same for most of us, we have got an idea of what that is' (FG1, P2, L70).

Participants asserted that compassion was communicated through the use of good interpersonal skills such as active listening and the use of empathy:

'[...] if you're just doing tasks [...] you're not actually realising that that person's probably been through two world wars [...] the person may never have been outside their home for the last 6 years, and you're the last person they've come across' (FG3, P2, L60).

Similarly, empathy is communicated through active listening, conveying an acknowledgment of the patient as a person with values and beliefs:

'[Compassion is demonstrated] by acknowledging [the patient's] values [...] listening to them.' (FG3, P1, L63, 65).

Ultimately, fundamental nursing is dependent on the interconnectedness of care and compassion, which are underpinned and enhanced through the maintenance of 
dignity and respect:

'[Fundamental nursing implies] to build on other things as care, [such as] dignity [and] respect.' (FG5, P6, L73).

There was a definite stance that fundamental nursing is about achieving 'whatever the patient needs'. While this appears at odds with the aforementioned complexities determining fundamental nursing, there appeared to be a consensus that fundamental nursing involved the outward behaviour of nurses ('nursing demeanour'), which was interpreted through the notion of 'care' and 'caring'.

Nursing demeanour: 'Care' and 'caring'. Key to the notion of fundamental nursing were participants' considerations of how nurses approach their daily work. One participant considers that 'care' can be interpreted as a functional (tasks) concept, but that 'caring' concerns an interpersonal concept manifested through the outward behaviour of the nurse:

'[...] there are [tasks nurses] have to do. But [fundamental nursing is] the extra bit [...] so you can come in be your task allocation nurse [...] but it's the bits in-between, so when you're doing the observations, talking to your patients [...] that's the caring side of it [...] the extra bits you do around the 
tasks, not just the tasks [...]' (FG3, P2, L54).

Not only is there a distinction between 'care' and 'caring' but also compassion in the way a nurse communicates with patients, demonstrating an authenticity, and care partnership. Ultimately:

'[...] the [nurse's] general approach would be the same, it should be welcoming, warming, professional, settled in your mind, as much as trying to get a grasp of the whole situation.' (FG2, P1, L115).

Nursing values

The synergy between the values participants held, how these values translated into fundamental nursing, and the subsequent nursing values participants considered, was evident through the $6 C s$.

6Cs: 6Cs 'encapsulate' nursing values. Participants across all focus groups agreed that the 6Cs neatly 'encapsulated' nursing values:

'[...] the 6Cs encapsulate all [nursing] values [...] take each element of the 6Cs and drill down a little bit further on it, but they are headlines.' (FG6, P2, L25). 
Although many participants could not recall every one of the 6Cs, they recognised each $6 \mathrm{C}$ was interdependent:

'If you decide that one day out of those 6Cs you are not going to be compassionate, then the rest of the 5Cs fall apart at the wayside. They are worthless without it.' (FG2, P1, L30).

The effect of the 6Cs must have had an impact on the above first-year nursing student, and perhaps illustrates the emphasis of developing a culture of compassionate care (NHS England, 2012) within nursing curricula. For other first-years, there was consideration of the 6Cs co-existing with the Nursing and Midwifery Council (NMC) Code (2015):

'I would expect [people of trust] to have a certain set of values and they should say values like The Code.' (FG5, P2, L20).

Having a code to follow was evidenced in other transcripts, whereby participants considered that nurses are 'signing up' to a profession and have a responsibility to adhere to professional standards: 
'[...] if you were a nurse then I think there's some core values that you need to uphold, whether you feel you agree with them or not, they are what you are signing up to uphold as your role as a nurse. So, whether that be the 6Cs, or other ones [...].' (FG1, P2, L3).

Although the 6Cs were considered by participants as useful in helping them to 'define core nursing values' (FG3, P1, L79), there was the contrary notion that even without the $6 \mathrm{Cs}$ existing as a strategy, the principles of the $6 \mathrm{Cs}$ would remain 'innate' for nurses and be applied tacitly in clinical practice:

'[Prior to the launch of the 6Cs] I think that [they] are innate. I don't think it makes a good nurse or a bad nurse, but I thinking [the 6Cs] are something we have inside us.' (FG4, P2, L51).

'[...] as a nurse, or a student nurse, we'd still, follow the [6Cs] [...] naturally because that is what we want to do like looking after someone, making sure it is compassionate, all of those, we would still follow them even if [the 6Cs were not] written down as the 6Cs, we would still do it.' (FG6, P3, L9).

6Cs: Compassion. Participants' linked compassion with the values of maintaining respect and dignity: 
'[...] you have to respect patient choices [...] their values and their ethics, whether you agree with them, or not' (FG1, P2, L3).

Participants questioned whether compassion was something that one could 'learn' or 'develop', such that the nursing student needed to exhibit a degree of compassion prior to nurse education:

'[...] "can you teach compassion[?]" [...] you should have some compassion ingrained in you already, otherwise why would you want to do the [nursing] course[?]' (FG4, P1, L52).

The above third-year nursing student's excerpt was shared with a first-year nursing student in another focus group, explaining:

'[...] that there has to be desire within you that's capable of putting that person before yourself and I think that's what possibly drives people to come to nursing' (FG5, P1, L54).

Whether compassion needs to be evident prior to a person commencing nurse education, or developed during the programme of study, an important element 
of authenticity and sincerity was evident:

\begin{abstract}
'[...] you have to be able to actually look somebody in the eye and show compassion [...] appropriate touch where it is needed [...] being able to show that you actually care and you are actually compassionate' (FG1, P2, L43).
\end{abstract}

\title{
Discussion
}

Findings highlighted the interrelationship between how participants understood the notion of values, such that values in general were inseparable from values in nursing. Although participants may have provided socially desirable answers before their peers and the facilitators, the concordance between their personal and professional values appears reassuring. The same was also true of participants having relatively consistent considerations regarding fundamental nursing and nursing values across the three years of nurse education, reflecting findings of Pitt et al. (2014) suggesting that personal qualities remain consistent. This was evidenced not only in the transparency between personal and professional values, whereby participants incorporated their personal values and life experiences within nursing, but through the notion of values as being a code (or ideals) of behaviour with respect and dignity being a fundamental ideal. Personal and professional value concordance reflects Drayton and Weston's (2015) findings that registered nurses 
share a passion for nursing and recognise that each nurse brings core values and individual ways of working with patients.

\section{Respect and dignity}

The interrelationship between respecting others and upholding an ethic of reciprocity was evident as both a general and nursing value, with complexity in delineating differences between respecting and caring for others. Kelly (1991) found that undergraduate nursing students understand professional values in nursing by having respect for others and taking time for little things, for example, in ensuring a patient is comfortable (Chipman, 1991). Specifically, respect is a broad phenomenon, encompassing aspects of earning patient trust and confidence, respecting privacy, active listening, exemplifying a positive demeanour, providing patient choice, and being person-centred. Furthermore, the little things refer to seemingly unremarkable elements of care provision, for example, sharing their worries. Williams et al. (2016) present similar findings for healthcare professionals, supporting the little things in 'caring for' and 'caring about' (p. 788) patients, helping them challenge poor care and bring about positive change.

Internationally, respecting and caring appear to comprise the essence of nursing, whereby nurses commit themselves to maintaining human dignity and preserve 
humanity (Gregg and Magilvy, 2004; Horton et al., 2007; Pang et al. 2009; Perry, 2005; Shih et al., 2008). For example, participant 1 (FG1, L69) stated that a nurse would be caring and maintain the patient's humanity in the process of providing functional care. Pearcey (2010) highlights the distinctions between care and caring, the former referring to functional tasks required to deliver treatment, compared with the latter referring to the affective component of how nursing care is provided, as discussed by McSherry et al. (2012). Affective care was exemplified through the use of 'sitting down talking to patients' (Pearcey, 2010, p. 54).

\section{Care and compassion}

Horton et al. (2007) highlight that care is synonymous with nursing, however, this still does not clarify the concept. A participant in Pearcey's (2007) study highlighted that they 'did not feel comfortable with the expression "caring"' and could not determine 'what word [one] would use for caring' (p. 53). Metaphorical notions of caring and compassion exist, with Pang et al. (2009) suggesting that care and compassion are interdependent when a nurse demonstrates, with a 'warm heart' (p. 314), a willingness to meet a patient's needs. This goes beyond the provision of completing a nursing task, and depends on keeping the patient, rather than the disease/diagnosis, central to care delivery (Shih et al., 2008). This reflects the ways nurses connect with patients by 'affirming the value of the person' (Perry, 2005, p. 
43), such that despite how unwell patients are, nurses continue to uphold the patient's sense of worth by using compassion to inform the patient they are more than a disease or diagnosis. Ensuring that compassion remains a priority in the delivery of care depends, in part, on a healthcare culture stipulating its values and ensuring these values enable staff to feel part of a group (Muls et al., 2015). A failure to do so might manifest as an organisational culture that is passive, habituated, sceptical, closed, and target-driven (Muls et al., 2015).

$6 C s$

The 6Cs (DH, 2012a; NHS England, 2012) pervaded throughout all participants' accounts of nursing values. There was a complexity in respect of participants needing to refer to the $6 \mathrm{Cs}$ to communicate nursing values, but state that even if the 6Cs were not coined, nurses would adopt the same values. Bostock-Cox (2013) highlights the oddity (and compassion fatigue) of nurses having trained before the 6Cs in needing to express principles considered implicit to the profession, and that they are embedded within the notion of emotional intelligence (Goleman, 1995). Compassion fatigue concerns the notion that nurses are prevented from caring in favour of meeting targets and reducing budgetary pressures, and can be referred to as the $6 B s$ : bullied, burnt out, bruised, blocked, bemused, and bewildered (Cummings, 2012). 
The notion that participants considered the $6 \mathrm{Cs}$ as innate, and that they would naturally follow even if not stated as ' $6 \mathrm{Cs}$ ', warrants further investigation. Because the $6 \mathrm{Cs}$ are introduced to nursing students as a fundamental part of their curriculum, it was not surprising to see these feature as core elements of participants' understanding of nursing values and fundamental nursing. However, the sustainability of the 6Cs depends on the extent to which they are embedded in nursing practice, with projects focused on promoting the application of the $6 \mathrm{Cs}$ across health and social care (Beal, 2014). Furthermore, it has been highlighted continued investment in staffing is required to sustain the $6 \mathrm{Cs}$ in practice (Scott, 2013).

\section{Limitations and recommendations for further research}

Cross-sectional methodologies only illustrate participant responses at one period in time. A longitudinal study would be required to determine qualitative changes for participants during nurse education.

Future studies examining factors that shape nursing students' values, attitudes and perceptions of compassionate care might focus on constructs of spirituality. It was interesting to note the spiritual dimension of holistic care was often forgotten about, 
and psychological care was not prioritised as much as attending to physical needs. Additional research is required to explore these complexities, not only regarding the delivery of care to patients, but also the training implications for nursing students in providing psychological and spiritual support for patients.

Exploring the development of nursing students' values would be strengthened considerably through employing a longitudinal design tracking a cohort of nursing students from the start of nurse education, through to completion, and ideally for a period of time post-registration.

\section{Conclusion}

There was considerable transparency between personal and professional values of participants in the way they would care with dignity, respect and compassion. The notion of fundamental nursing, whilst complex to determine, ran concurrent to core nursing values which were exemplified through the 6Cs. Participants valued the opportunity of sharing their thoughts on fundamental nursing and nursing values, and affirmed this was as important for their personal and professional development, as was developing professional competency in clinical nursing. 


\section{References}

Beal J (2014) Embedding the 6Cs across health and social care. Nursing Times 110(49): 22-23.

Biro AL (2012) Creating conditions for good nursing by attending to the spiritual. Journal of Nursing Management 20(8): 1002-1011.

Bostock-Cox B (2013) The 6 Cs: Are they relevant to practice nursing? Practice Nurse 43(9): 10-13.

Bradshaw A (2011) Compassion: What history teaches us. Nursing Times 107(1920): $12-14$.

Bradshaw A (2015) An analysis of England's nursing policy on compassion and the 6 Cs: The hidden presence of M. Simone Roach's model of caring. Nursing Inquiry 23(1): 78-85.

Bramley L and Matiti M (2014) How does it really feel to be in my shoes? Patients' experiences of compassion within nursing care and their perceptions of developing compassionate nurses. Journal of Clinical Nursing 23(19-20): 2790-2799.

Braun V and Clarke V (2006) Using thematic analysis in psychology. Qualitative Research in Psychology 3(2): 77-101.

Care Quality Commission (2015) The state of health care and adult social care in England, 2014 to 2015. Available at: 
https://www.gov.uk/government/publications/health-and-adult-social-carein-england-2014-to-2015 (accessed 26 April 2016).

Chipman Y (1991) Caring: Its meaning and place in the practice of nursing. Journal of Nursing Education 30(4): 171-175.

Cummings J (2012) Developing a vision \& strategy for nursing, midwifery, and care-givers. Available at: http://www.hpft.nhs.uk/ uploads/documents/thetrust/equality-and-diversity/jane-cummings-keynote-speech.pdf (accessed 4 June 2016).

Curtis K, Horton K and Smith P (2012) Student nurse socialisation in compassionate practice: A grounded theory study. Nurse Education Today 32(7): 790-795.

Dean E (2012) Health professionals should be assessed for compassion. Nursing Older People 24(3): 6-7.

Department of Health (2012a) Compassion in practice. London: Department of Health.

Department of Health (2012b) Developing the culture of compassionate care: Creating a new vision for nurses, midwives and care-givers. London: Department of Health. 
Drayton N and Weston KM (2015) Exploring values in nursing: Generating new perspectives on clinical practice. Australian Journal of Advanced Nursing 33(1): 14-22.

Francis R (2013) Report of the Mid Staffordshire NHS Foundation Trust Public Inquiry. London: The Stationary Office.

Goleman D (1995) Emotional intelligence. London: Bloomsbury Publishing PLC.

Gregg MF and Magilvy JK (2004) Values in clinical nursing practice and caring. Japan Journal of Nursing Science 1(1): 11-18.

Horsburgh D and Ross J (2013) Care and compassion: The experiences of newly qualified staff nurses. Journal of Clinical Nursing 22(7-8): 1124-1132.

Horton K, Tschudin V and Forget A (2007) The value of nursing: A literature review. Nursing Ethics 14(6): 716-740.

Kelly B (1991) The professional values of English nursing undergraduates. Journal of Advanced Nursing 16(7): 867-872.

Keogh B (2013) Review into the quality of care and treatment provided by 14 hospital trusts in England: Overview report. London: NHS England.

McSherry W, McSherry R and Watson R (2012) Care in nursing: Principles, values and skills. Oxford: Oxford University Press.

Morris-Thompson T (2012) Students tested for compassion as well as academic ability. Nursing Older People 24(3): 5. 
Muls A, Dougherty L, Doyle N, et al. (2015) Influencing organisational culture: A leadership challenge. British Journal of Nursing 24(12): 633-638.

NHS England (2012) Our culture of compassionate care. Available at: https://www.england.nhs.uk/wp-content/uploads/2012/12/6c-a5-leaflet.pdf (accessed 3 June 2016).

Nursing and Midwifery Council (2015) The Code for nurses and midwives. Available at: https://www.nmc.org.uk/standards/code/ (accessed 3 June 2016).

Pang D, Senaratana W, Kunaviktikul W, et al. (2009) Nursing values in China: The expectations of registered nurses. Nursing and Health Sciences 11(3): 312317.

Patton MQ (1990) Qualitative evaluation and research methods. California: Sage.

Pearcey P (2010) 'Caring? It's the little things we are not supposed to do anymore'. International Journal of Nursing Practice 16(1): 51-56.

Perry B (2005) Core nursing values brought to life through stories. Nursing Standard 20(7): 41-48.

Pitt V, Powis D, Levett-Jones T et al. (2014) Nursing students' personal qualities: A descriptive study. Nurse Education Today 34(9): 1196-1200.

Scott G (2013) Equipment and staffing are vital to 6Cs' success. Nursing Standard 28(14): 3 . 
Shih FJ, Lin YS, Smith MC, et al. (2008) Perspectives on professional values among nurses in Taiwan. Journal of Clinical Nursing 18(10): 1480-1489.

Thorpe K and Loo R (2003) The values profile of nursing undergraduate students: Implications for education and professional development. Journal of Nursing Education 42(2): 83-90.

van der Cingel M (2014) Compassion: The missing link in quality of care. Nurse Education Today 34(9): 1253-1257.

Williams V, Kinnear D and Victor C (2016) 'It's the little things that count': Healthcare professionals' views on delivering dignified care: A qualitative study. Journal of Advanced Nursing 72(4): 782-790.

Willig C (2008) Introducing qualitative research in psychology (2nd Ed.). Maidenhead: Open University Press. 


\section{Key points}

- Compassion in care is highly topical arising out of compassion being the historical basis for nursing, being subject to scrutiny through recent failings of care.

- $\quad$ Nursing students understand the notion of values through the various factors influencing the development and refinement of values, such as culture, childhood upbringing and life experience.

- Nursing students demonstrate transparency between personal and professional values, placing respect and dignity key to both.

- Fundamental nursing is complex to determine, but best understood through the provision of care for 'whatever the patient needs' in a caring way.

- $\quad$ Nursing values are exemplified through the notion of the $6 \mathrm{Cs}$.

- $\quad$ Findings provide a useful platform for nursing students to reflect on their personal/professional values, and nurse educators to develop curricula to foster the affective (caring) skills of nursing students alongside clinical competence. 